\title{
Effect of Stress Field on Closure of Center Defects in Symmetric Rolling and Asymmetric Rolling of Round Billets ${ }^{* 1}$
}

\author{
Tatsuro Katsumura ${ }^{1, * 2}$, Takaaki Iguchi ${ }^{1}$, Hideto Kimura ${ }^{2}$ and Jun Yanagimoto ${ }^{3}$ \\ ${ }^{1}$ Steel Research Laboratory, JFE Steel Corp., 1-1, Kawasaki-cho, Handa 475-8611, Japan \\ ${ }^{2} J F E$ Techno-Research Corporation, Kawasaki 210-0855, Japan \\ ${ }^{3}$ Institute of Industrial Science (IIS), The University of Tokyo, Tokyo 153-8505, Japan
}

\begin{abstract}
Although the closure of defects at the center of billets on rolling such billets is an important subject, a method of quantity evaluation for such closure has not yet been clarified. Therefore, to establish a method for such quantity evaluation, we study such defects using experiments and finite element analysis including asymmetric rolling, especially with respect to the integration of the hydrostatic stress Gm. The results showed that stress was widely distributed, as determined in the experiment and in the FEM analysis of rolling at the edge around an artificial central hole in which defects were simulated. Therefore, it was suggested that, to evaluate the behavior of defect closure, the size of defects and the shape of the grooved roll used should be taken into account. [doi:10.2320/matertrans.P-M2014837]
\end{abstract}

(Received March 30, 2014; Accepted September 11, 2014; Published November 14, 2014)

Keywords: rolling, bar rolling, deformation behavior, stress field, defect, closure, hydrostatic stress, grooved roll, asymmetric rolling

\section{Introduction}

Steel materials offer many advantages. In addition to their diverse material properties, i.e., mechanical properties and corrosion resistance, numerous cross-sectional shapes can be manufactured relatively easily. For this reason, steel is used in many products, for example, automobiles and home electrical appliances, as well as a wide range of other applications from large-scale structures such as bridges, shipbuilding, etc. to electromagnetic materials used in miniature electronic equipment. In the manufacturing of these products, in many cases, slabs, billets, etc. are produced as semi-finished products, and hot rolling and other processes are then applied to those materials.

Slabs and other semi-finished products are produced from larger cast slabs or ingots by performing plastic working such as rolling or forging. Larger-scale cast slabs or ingots have advantages, as they respond to the trend toward larger-scale products and the dimensions of the semi-finished product can be produced as required for specific applications with relative freedom. On the other hand, however, due to the increased cross-sectional area, casting defects tend to be included or the size of those defects tends to increase. Thus, reducing these internal defects is an important issue.

When such internal defects are eliminated by forging, it is known that internal quality generally improves as the processing degree increases in closure or pressure bonding of defects. ${ }^{1)}$ However, if large reduction is applied with flat dies, the Mannesmann effect ${ }^{2}$ ) occurs in the material center, and this causes cracking, which reduces the closure effect. Therefore, the FM forging method, etc. ${ }^{3,4)}$ were developed to minimize this phenomenon, and the closure conditions, etc. in this process have also been discussed. ${ }^{4-7)}$ In rolling, it has been reported that application of heavy reduction is effective with heavy-gauge plates, etc. ${ }^{8-11)}$ In addition to increasing the processing degree, research has also shown that defects can

\footnotetext{
${ }^{* 1}$ This Paper was Originally Published in Japanese in J. JSTP 54 (2013) 612-617.

${ }^{* 2}$ Corresponding author, E-mail: t-katsumura@jfe-steel.co.jp
}

be closed more efficiently in rolling and forging processes by providing a temperature difference between the material surface and center. ${ }^{12)}$

Conventionally, this type of defect closure was mainly studied by experimental techniques. However, study of advantageous conditions for closure and their optimization using numerical analysis has also been performed. ${ }^{13,14)}$ Comparison of the results of deformation analyses by FEM and actual defect closure behavior is frequently used, particularly in forging, and based on reports that closure behavior can be explained by the stress field and strain field obtained from analysis, ${ }^{15,16)}$ quantitative evaluation of closure behavior by parameters has been attempted. ${ }^{17-19)}$

Methods of quantifying closure behavior in rolling include a technique for predicting closure from a velocity field ${ }^{20}$ ) and estimation of the closure effect by using the length of the contact arc between the roll and workpiece as a parameter, ${ }^{21)}$ a hydrostatic integration index, which is obtained by dividing hydrostatic pressure by equivalent stress and integrating the result by strain in forging analysis has been proposed, ${ }^{22)}$ and its application to rolling has also been studied. ${ }^{23)}$ Since the hydrostatic integral equation takes the same form as Oyane's criterion, ${ }^{24)}$ which is a well-known equation expressing ductile fracture, it can be said that the hydrostatic integral equation expresses the closure of defects due to volumetric strain caused by deformation. If this equation is compared with actual phenomena, it shows the same tendencies as the knowledge concerning heavy reduction rolling, that is, high hydrostatic pressure is effective for defect closure. Therefore, this equation is considered capable of expressing phenomena with good accuracy and has also been applied to study of actual manufacturing processes. $^{23)}$

As a distinctive feature of the technique in which study is performed using this equation, the stress field at the location where a defect exists is obtained by analysis; that is, in many cases, the material center is obtained and used for the study. However, in obtaining the stress field, the general practice is to use the results of an analysis of an object without a material defect, namely, a solid material. Hence, the possible 
difference between this result and the stress field of an actual member containing a defect is an essential concern.

Therefore, in this report, the stress field in a billet center was compared by analysis and rolling experiments, and quantitative evaluation of closure in materials containing defects was attempted. The following presents the results of this research.

\section{Experimental Method}

\subsection{Verification of accuracy of FEM analysis of rolling stress in material center}

One method of measuring the stress field generated in the material center during rolling is direct measurement by arranging sensors such as pressure gauges in the center. ${ }^{25}$ ) First, therefore, the stress at the material center obtained in a rolling experiment with solid material and by FEM analysis was compared, and the accuracy of the FEM analysis was verified.

The comparison of the stress obtained by FEM analysis and by actual measurement was performed by rolling a plasticine test piece which had been prepared with a roundbar shape. The prepared test piece was cut longitudinally along the material axis, stress sensors were embedded in the center in three directions (reduction direction, direction orthogonal to the reduction direction, and rolling direction), and the test piece was then restored to the original round-bar shape. The test piece is shown schematically in Fig. 1. Rolling was performed using diamond- and flat-shaped calibers in a room held at a constant temperature. As rolling conditions, the billet diameter was $\varphi 80 \mathrm{~mm}$, the roll diameter of the flat roll was $\varphi 140 \mathrm{~mm}$, and the diameter of the flange part of the roll with the diamond-shaped caliber shown in Fig. 2 was $\varphi 170 \mathrm{~mm}$. The caliber dimensions were adjusted by the flange diameter ratio of the rolls used. The amount of rolling reduction height was controlled at $15 \mathrm{~mm}$ by tightening the roll gap.

\subsection{Study of closure behavior of defect in billet center}

It is difficult to apply the direct measurement method described in the previous section to measurement of the stress field/strain field around a small defect in the material center. Moreover, in the comparison with FEM analysis, which is generally performed, accurate estimation of the stress distribution and strain distribution in the vicinity of the defect is also difficult, even if a rough estimation from its deformation is possible. Therefore, as the method used in this report, a center defect was introduced artificially, and slits were made around its periphery. Rolling experiments were performed assuming the strain field and hydrostatic stress distribution can be expressed by the closure behavior after rolling, and at the same time, the results were compared with an FEM analysis.

The slit material closure experiment was performed by hot rolling. The rolling material was SUS420J1, which has a comparatively low rolling load, shows little scale effect, and is easy to handle. As shown in Fig. 3, the outer diameter of the test piece was $50 \mathrm{~mm}$, and a $\varphi 5 \mathrm{~mm}$ hole was made as a center defect. Slits with a width of $0.3 \mathrm{~mm}$ and length of $2 \mathrm{~mm}$ were made around the edge of the hole in 8 directions

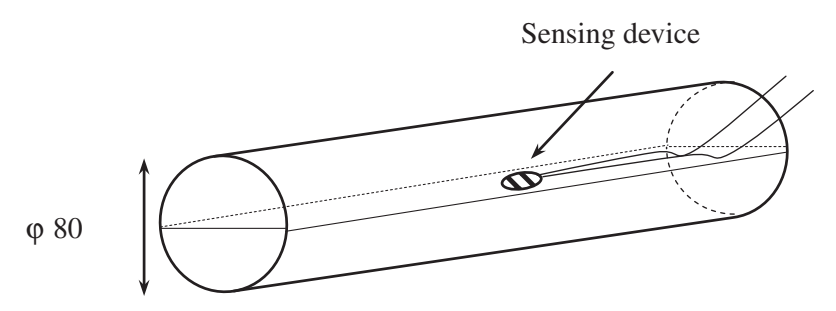

Fig. 1 Measuring method in caliber rolling at billet center using plasticine.

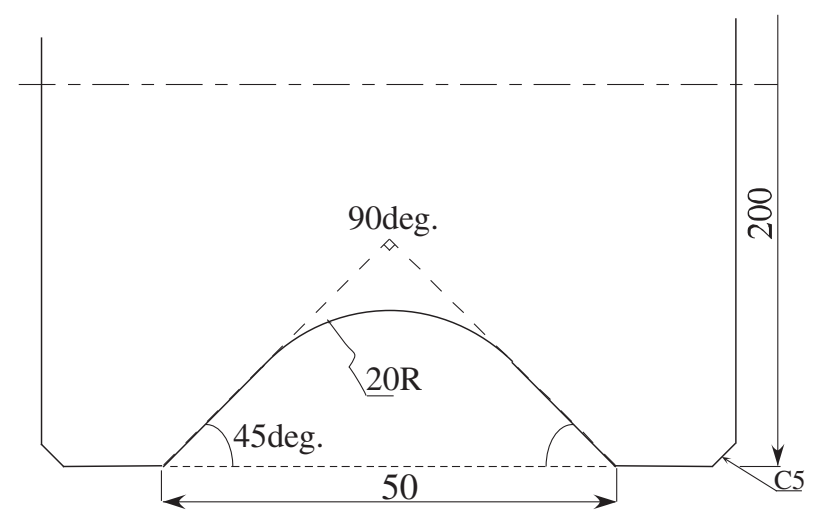

Fig. 2 Roll and caliber shape.

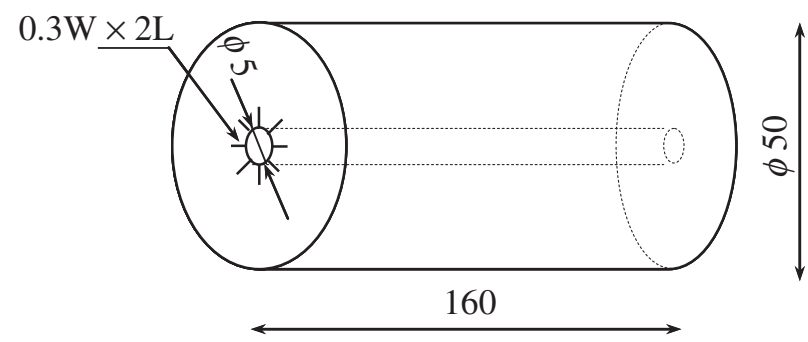

Fig. 3 Shape of test piece.

at a pitch of $45^{\circ}$ by electro discharge machining (EDM). The test piece was heated to $1200^{\circ} \mathrm{C}$ in an electric furnace while simultaneously blowing nitrogen, and rolling was performed at various rolling reductions with rolls having a diameter of the flange for $200 \mathrm{~mm}$, on which diamond-shaped calibers had been machined.

\subsection{FEM analysis solver and analysis conditions}

The FEM analysis was performed with the rigid-plastic finite element analysis solver CORMILL, ${ }^{26)}$ which uses the method of Lagrange multiplier. Then, as the stress field used when arranging the results, in the case of rolling material with a round cross-sectional shape with the diamond-shaped caliber, the value given at the cross section of the roll-bite dead-point was used because stress reaches its maximum in the vicinity of the exit side of the roll bite. Considering vertically asymmetrical rolling, a half cross section was used. In this cross section, the mesh was divided into 10 meshes in the radial direction and 18 in the circumferential direction. In the longitudinal direction, a mesh of approximately 10 was used in the roll bite, and deformation regions were provided on the entry and exit sides. Using this mesh, a 3-dimensional analysis was performed by 8 -node hexahedral isoparametric reduced integration elements. 
Flow stress $\sigma_{\text {eq }}$ was given as deformation resistance. This was obtained by the mean deformation resistance equations of Inoue ${ }^{27)}$ and Misaka ${ }^{28}$ from the results of a uniaxial compression experiment with the material used in these experiments. The temperature distribution was assumed to be uniform in the cold condition. In the hot condition, based on the results of temperature measurements, the surface layer temperature was set at $1100^{\circ} \mathrm{C}$ and the internal temperature at $1200^{\circ} \mathrm{C}$, which was the same as the heating temperature. Regarding the rolls, the coefficient of friction was set at 0.25 for cold rolling and 0.5 for hot rolling under the Coulomb friction condition. A rolling analysis was also performed for a hollow material with an outer diameter of $\varphi 50 \mathrm{~mm}$, simulating the material center defect, and a quantitative evaluation of closure by the obtained stress/strain was discussed.

As rolling types, in the following figures, rolling with a pair of flat rolls without calibers is denoted by F-F rolling (flat rolling), rolling with a pair of diamond calibers is denoted by D-D rolling (diamond rolling), and rolling with a combination of a flat roll and a diamond caliber, in which rolling is asymmetrical, is denoted by F-D rolling (flatdiamond rolling).

\section{Experimental and Analytical Results and Study}

\subsection{Quantitative evaluation of FEM analysis by rolling experiment with solid material}

In order to verify the appropriateness of the stress field obtained from the FEM analysis, solid material was rolled with a caliber shape in which the diameter ratio of the rolls used and the flange in Fig. 2 was used as a scale factor, and the results were verified by comparison with those of direct measurement.

The stresses obtained in the experiment and FEM analysis were normalized by the absolute value of stress in the reduction direction in flat rolling, $\sigma_{y}$. The results are shown in Fig. 4. Based on these results, generally good agreement was confirmed between the analytical and experimental stress fields from comparisons of $\sigma_{x}$ and $\sigma_{y}$ in both the cases of flat rolling and diamond rolling. Accordingly, in the following, the stress field obtained by FEM analysis under the same conditions as in experimental hot rolling is treated as approximately identical with that in actual hot rolling.

\subsection{Slit closure behavior in diamond rolling}

A test piece with a hole and slits as an artificial defect was rolled using diamond calibers. In this hot rolling, an experiment was performed with $2 \mathrm{~mm}$ as the target rolling reduction of the outer diameter of the plane between the opposed upper and lower calibers. An FEM analysis was also performed using the same calibers and rolling reduction, and the cross section and shape sampled from the longitudinal center of the test piece after rolling were compared with the analytical results. Because it was difficult to judge the slit closure behavior as such from the analytical results, a mesh containing only an artificial defect was prepared, and the stress field at the edge of that hole and the slit closure in the experiment were compared.

The results are shown in Fig. 5. From this figure, good agreement can be observed between the analytical wire frame

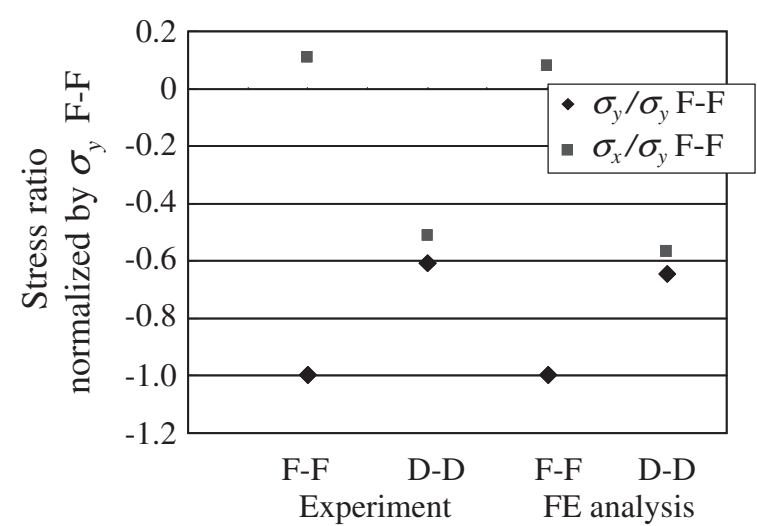

Fig. 4 Comparison of experiment with analysis of Flat-Flat/Dia.-Dia. rolling.

and the outer shape of the colored material after rolling and closure condition of the vacancy in the billet center. Nonclosure of the slits in the experimental material can also be confirmed. Regarding this non-closure, although the hydrostatic stress $\sigma_{\mathrm{m}}$ (also shown in Fig. 5) is compressive, its value is less than $50 \mathrm{MPa}$, which is small considering the fact that the deformation resistance of SUS420J1 at $1200^{\circ} \mathrm{C}$ under strain of approximately the same level is $75 \mathrm{MPa}$. Therefore, it is considered that the slits did not close.

The results when the diameter reduction of the same material was $5 \mathrm{~mm}$, or 2.5 times that in the previous experiment, and reduction was increased by $15 \%$ as reduction in area were arranged in the same manner. The shape comparison and distribution of hydrostatic stress obtained by FEM analysis are shown in Fig. 6. In the experimental results, the slits were substantially closed at the hole edge. On the other hand, comparing the analytical results in Fig. 5 and Fig. 6, because the increase in rolling reduction also increased hydrostatic stress over $50 \mathrm{MPa}$, and the increase occurred on the compressive side up to a value roughly approaching deformation resistance. It is considered that the slits closed due to this effect, thus confirming a strong correlation between the stress field and slit closure behavior.

Figure 7 shows the results of a comparison of the hydrostatic stress generated at the hole edge standardized for the flange part in the cross section at the exit side of the roll bite at the two different levels of rolling reduction discussed above. In this figure, the positive angles show the top roll side, and the negative values show the bottom roll side. From these results, it can be understood that apparent slit closure has occurred in a compressive field with hydrostatic stress of $42 \mathrm{MPa}$ or higher, and the slits remain open at compressive stress values of $30 \mathrm{MPa}$ or less.

\subsection{Slit closure behavior in vertically asymmetrical rolling with flat roll and diamond caliber}

The same study was carried out by flat-diamond rolling. The shape comparison and hydrostatic stress distribution are shown in Fig. 8. As distinctive results obtained by this rolling experiment and analysis, slit closure occurred only in the horizontal and lower $45^{\circ}$ directions, and the areas with a strong compressive tendency were also similar to this in the analysis. Because vertically asymmetrical rolling causes 

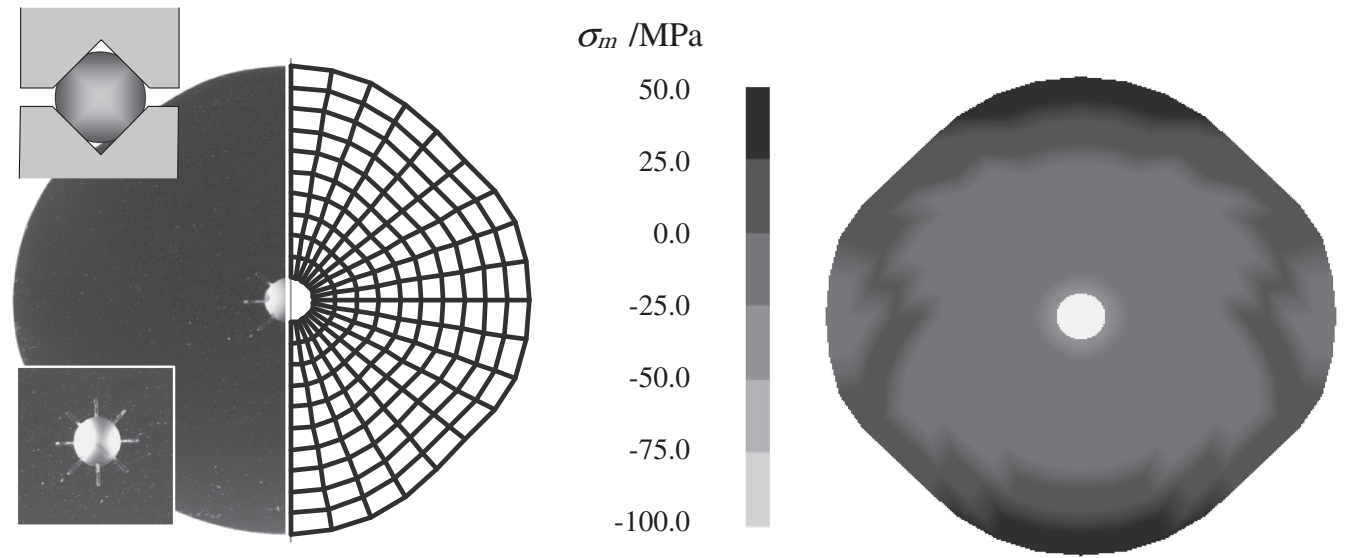

Fig. 5 Comparison of experiment with analysis of small reduction Dia. rolling, contour of mean stress (diameter reduction 2 mm).

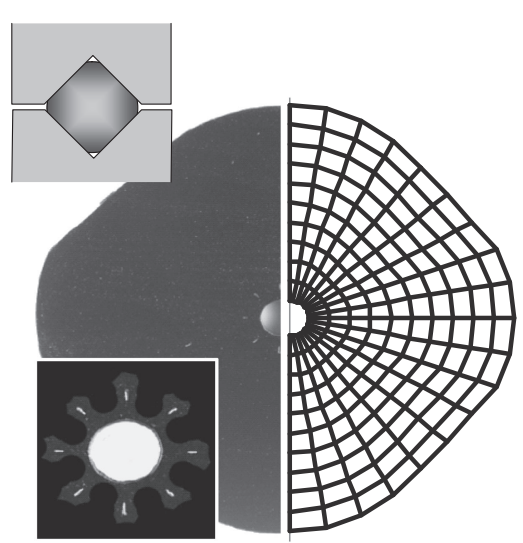

$\sigma_{m} / \mathrm{MPa}$

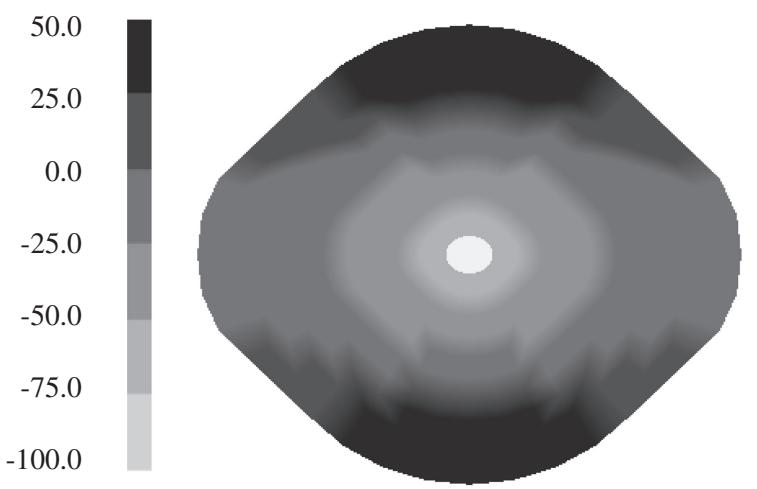

Fig. 6 Comparison of experiment with analysis of Dia. rolling, contour of mean stress (diameter reduction $5 \mathrm{~mm}$ ).

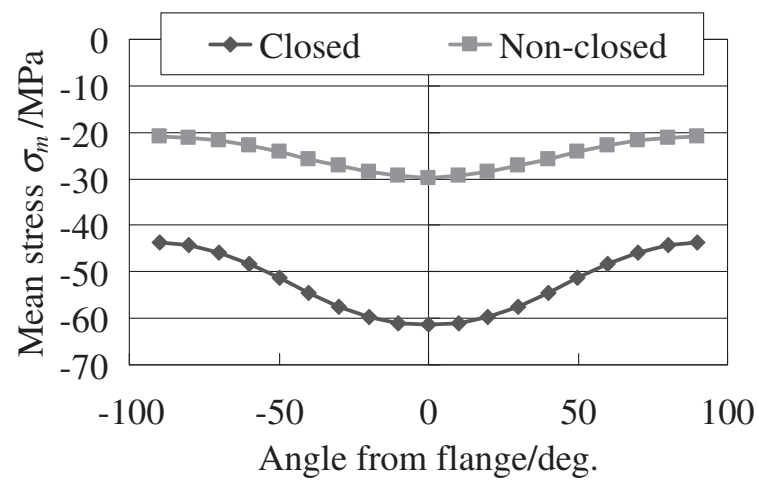

Fig. 7 Relationship between mean stress and angle from flange in Dia. rolling.

camber, the experiment and analysis were performed while varying the material inlet height. Figure 9 shows the results of a comparison of this stress field at the hole edge with that in diamond rolling. Comparing the closure behavior with the hydrostatic stress distribution in Fig. 9, the points of closure in the horizontal direction and the opening tendency in the downward direction can be expressed well. Although some unclear points concerning closure in the lower $45^{\circ}$ direction remain from the viewpoint of the consistency of the threshold value with that in diamond rolling, it was found that the asymmetry of stress distribution can be expressed satisfactorily by using the closure behavior of the slits.
These results clarified the facts that it is possible to estimate stress fields by FEM analysis based on slit closure behavior, and an asymmetrical stress field occurs also at the edge of an artificial hole in the center of a workpiece in asymmetrical rolling.

\subsection{Distribution of hydrostatic integration parameter, $\mathrm{Gm}$, and stress distribution in asymmetrical rolling}

This research revealed that the stress in the vicinity of a defect has a distribution and that distribution is remarkable in asymmetrical rolling. Therefore, the distribution of the hydrostatic integration parameter $\mathrm{Gm}^{22}$ (hereinafter, $\mathrm{Gm}$, $\sigma_{\mathrm{m}}$ represents hydrostatic stress and the positive values show tensile stress) shown by eq. (1) was obtained along a streamline at positions developed in the circumferential direction from the stress field at the edge of a $\varphi 5 \mathrm{~mm}$ hole in the cross section at the exit side of the roll bite and the strain history in the roll bite. And the result was compared with the $\mathrm{Gm}$ in the billet center obtained from the results of an FEM analysis of rolling of a solid material when similar reduction of the outer diameter was performed. The results are shown in Fig. 10. In this figure, the results for the solid material are shown at the $0^{\circ}$ position.

$$
\mathrm{Gm}=\int \frac{\sigma_{\mathrm{m}}}{\sigma_{\mathrm{eq}}} d \varepsilon_{\mathrm{eq}}
$$




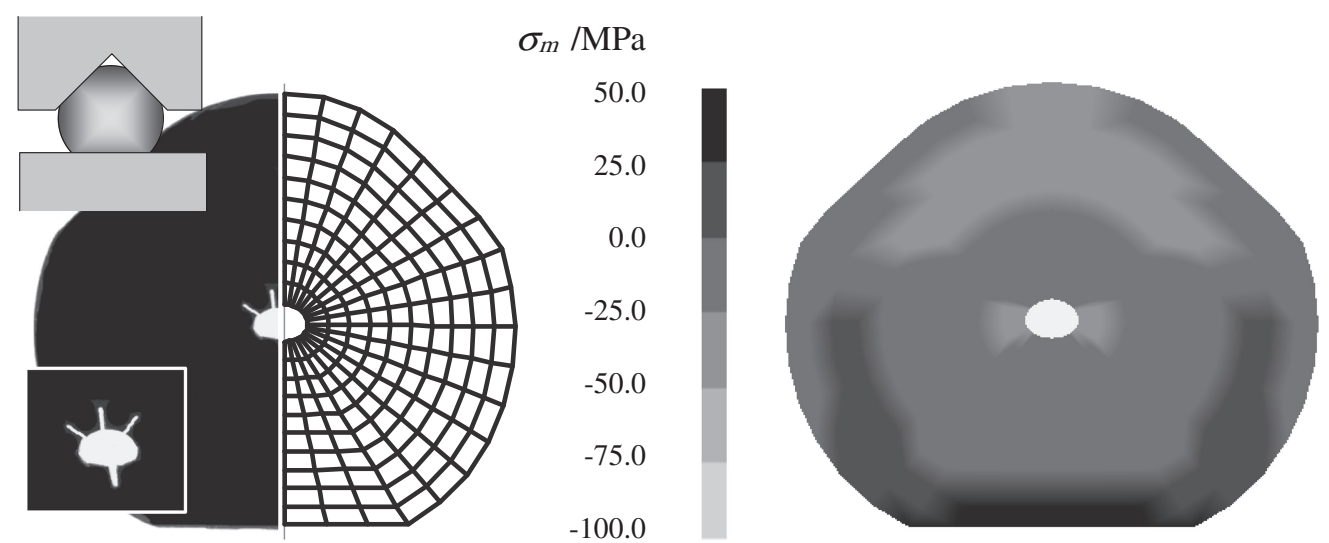

Fig. 8 Comparison of experiment with analysis of Dia.-Flat rolling, Contour of mean stress.

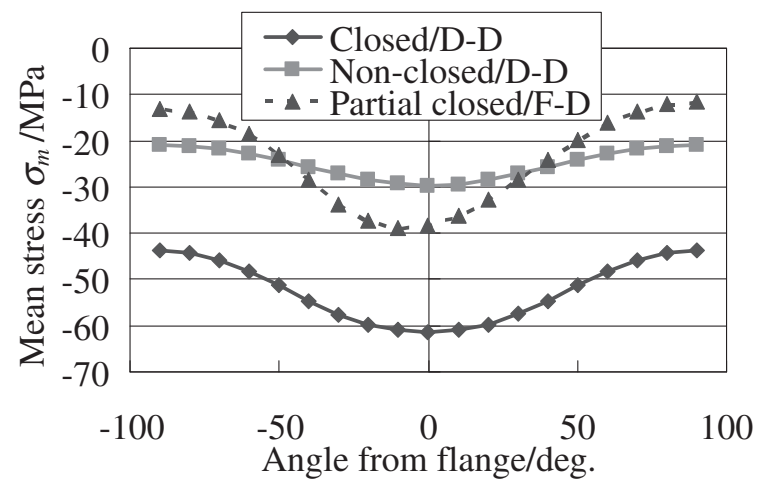

Fig. 9 Relationship between mean stress and angle from flange in Dia.-Dia./Flat-Dia. Rolling.

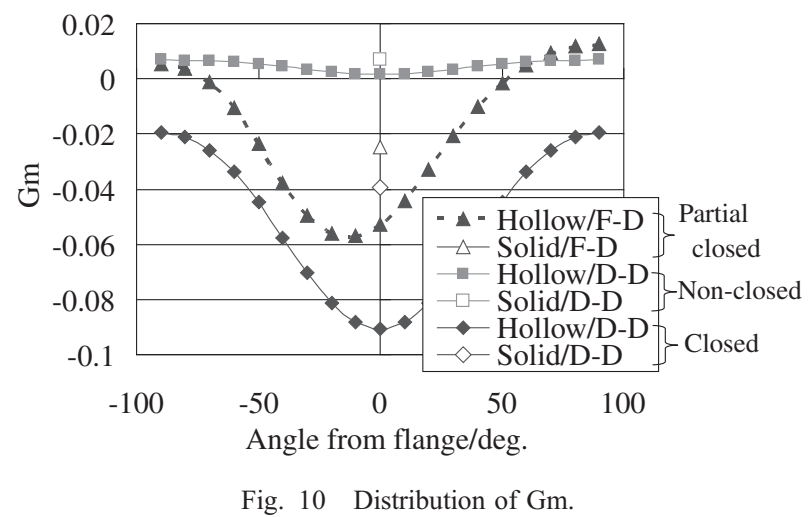

From this figure, the circumferential distribution of $\mathrm{Gm}$ at the defect hole edge has a wide distribution when reduction exceeds a certain value. Further, assuming closure behavior can be evaluated from this, $\mathrm{Gm}$ in case a defect is not considered, which is frequently used in conventional practice, is within the maximum and minimum values of $\mathrm{Gm}$ at each node when a defect is considered, excluding cases of small reduction, and there is a high possibility that its value depends on the distribution of $\mathrm{Gm}$ around the defect, including in asymmetrical rolling.

Accordingly, in application of $\mathrm{Gm}$, which is considered to be effective for predicting the closure behavior of material defects, there is a possibility of inconsistency between the stress distribution of an actual member with a defect and the results of analysis of a solid material, depending on the

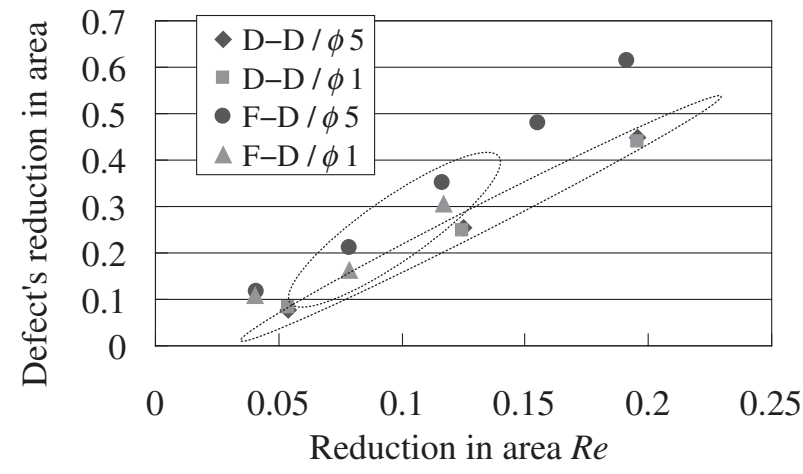

Fig. 11 Relationship between bulk reduction and defect reduction in area in Dia.-Flat/Dia. Rolling (FEM).

rolling conditions. Thus, the necessity of studying application of Gm, carefully considering that stress distribution, was suggested.

\subsection{Effects of defect diameter and rolling type on $\mathbf{G m}$}

The fact that the stress/strain fields in the vicinity of a defect control its closure behavior is considered to be unquestionable, including the effect of hydrostatic pressure.

However, the stress state around a defect should differ depending on the initial defect size. Therefore, the effect of the defect diameter and rolling type on Gm was studied by an FEM analysis. In this analysis, Gm was calculated from the stress obtained at various reductions in area, $R e$, of the bulk, which was controlled by changing the roll gap, and the results were also compared with the reduction in area of the defect.

The relationship between $\mathrm{Re}$ of the bulk and reduction in area of the defect was investigated by rolling analyses of diamond rolling and flat-diamond rolling. This is shown in Fig. 11. When using only diamond calibers (i.e., diamond rolling), the result was independent of the defect diameter within the range studied in this research, and defect reduction in area also increased in approximately the same manner with the increase of $R e$ of the bulk. On the other hand, in asymmetrical flat-diamond rolling, the tendency of defect reduction in area became stronger as the defect size increased. In explaining this result, width spread is slight ${ }^{29)}$ and the material displays a strong tendency to elongate comparatively uniformly in diamond rolling; therefore, it is considered that defect reduction in area has a strong 


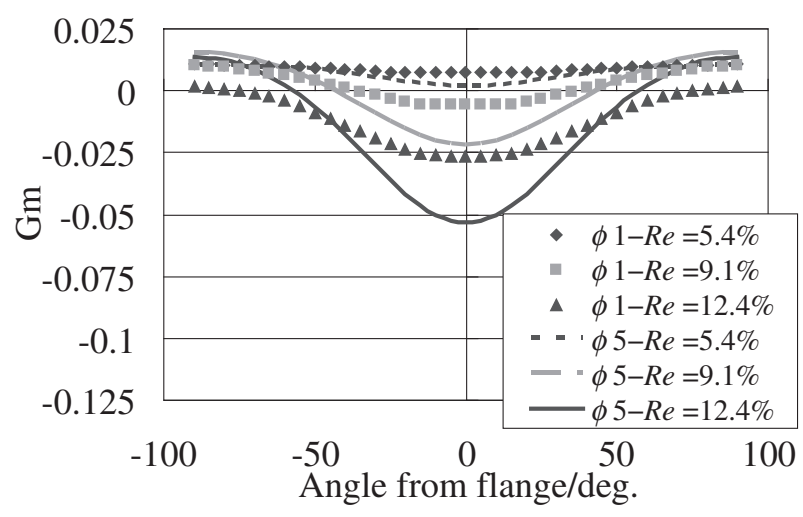

Fig. 12 Effect of defect diameter in Dia. Rolling (FEM).

correlation with $R e$ of the bulk and does not depend on the defect size. In contrast, in asymmetrical rolling with flat roll on one side, it is estimated that cross-sectional elongation of the material is non-uniform, depending on the position. In other words, the non-uniformity of the stress-strain field becomes stronger, and as a result, reduction of the defect is influenced by its size.

Therefore, next, the results of the FEM analysis of diamond rolling were evaluated focusing on Gm around the vacancy. This is shown in Fig. 12. In the case of light rolling reduction, the circumferential distribution of $\mathrm{Gm}$ is small and shows virtually no dependency on the defect diameter. However, when rolling reduction is increased, differences in the circumferential direction become large, depending on the defect size. Therefore, Gm at the nodes shown in Fig. 12 was averaged in the circumferential direction, and an additional FEM analysis of the heavy reduction side was performed. A comparison of the average of $\mathrm{Gm}$ and defect reduction in area using these results is shown in Fig. 13. In this figure, 2 plots with virtually the same values exist at each point on the $\mathrm{x}$-axis. These are plots of the results for different defect sizes at the same reduction in area. In diamond rolling at the same reduction in area, the initial defect diameters have hardly any effect on the average of $\mathrm{Gm}$ in the circumferential direction, and the corresponding defect reduction in area is approximately the same. In other words, as can also be understood from Fig. 11, defect closure depends on the reduction in area of the bulk and is not significantly affected by defect size.

The same study was carried out for the case of asymmetrical flat-diamond rolling. In comparison with the diamond rolling discussed above, a non-uniform distribution in the circumferential direction like that in the results studied in the previous section can be observed. This is shown in Fig. 14. The relationship between average $\mathrm{Gm}$ and defect reduction in area is shown in Fig. 15. In Fig. 15, the average of $\mathrm{Gm}$ at the same bulk $\mathrm{Re}$ and different defect sizes corresponds, for example, to approximately -0.37 and -0.45 . Unlike diamond rolling, in flat-diamond rolling, the average of $\mathrm{Gm}$ differs depending on the defect diameter even at the same Re, and the average of Gm displays a high correlation with defect reduction in area. Regarding the dependency of the average of $\mathrm{Gm}$ on defect size, it is estimated that it is largely due to the change of extensional behavior because rolling is performed with a combination of a diamond caliber and a flat roll, and the resulting effect.

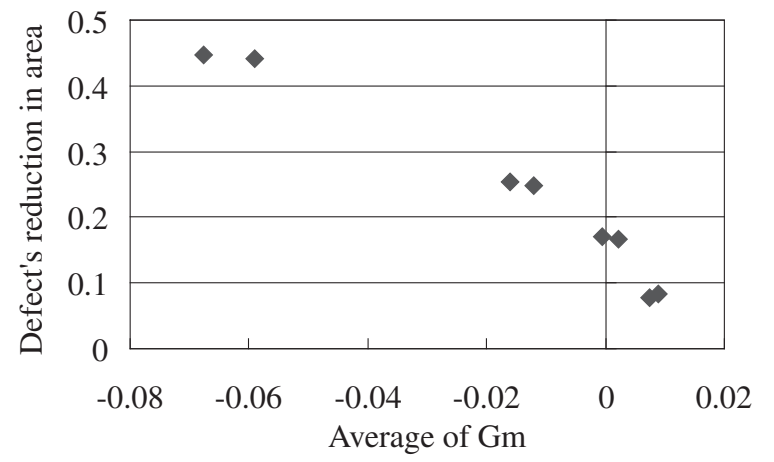

Fig. 13 Relationship between average $\mathrm{Gm}$ and defect reduction in area in Dia. rolling (FEM)

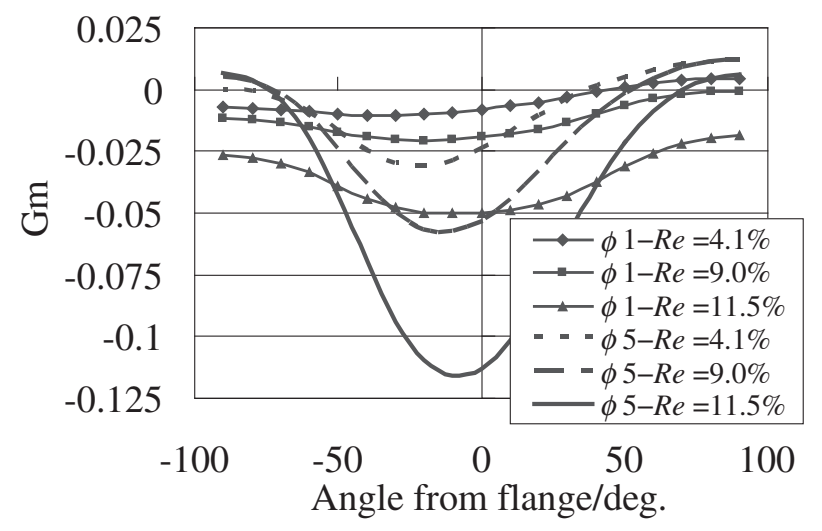

Fig. 14 Effect of defect diameter in Dia.-Flat rolling (FEM).

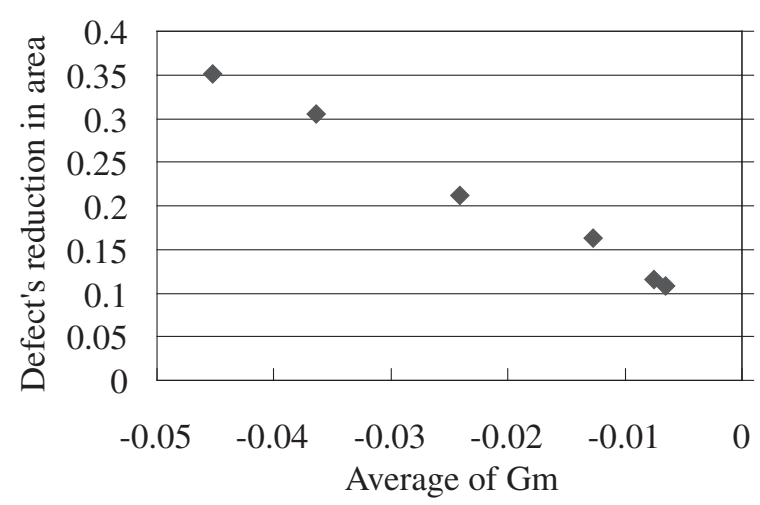

Fig. 15 Relationship between average $\mathrm{Gm}$ and defect reduction in area in Dia.-Flat rolling (FEM).

As described above, it became clear that the effect on defect closure differs depending on the defect diameter and rolling type. Therefore, the relationship between this difference in defect size and the average of Gm was studied for a cross-sectional reduction in area of approximately $9 \%$. The results were also compared with those for a solid material. This is shown in Fig. 16.

As with the results in Fig. 13, in diamond rolling, the distribution of the average of Gm only changes within a small range relative to changes in the defect diameter, whereas, in flat-diamond rolling, changes in the average of Gm depending on differences in defect diameter could be clearly observed. Here, the results for the solid material shown in Fig. 10 correspond to the case of a defect diameter of $0.0 \mathrm{~mm}$. Therefore, with both rolling types, the values fall 


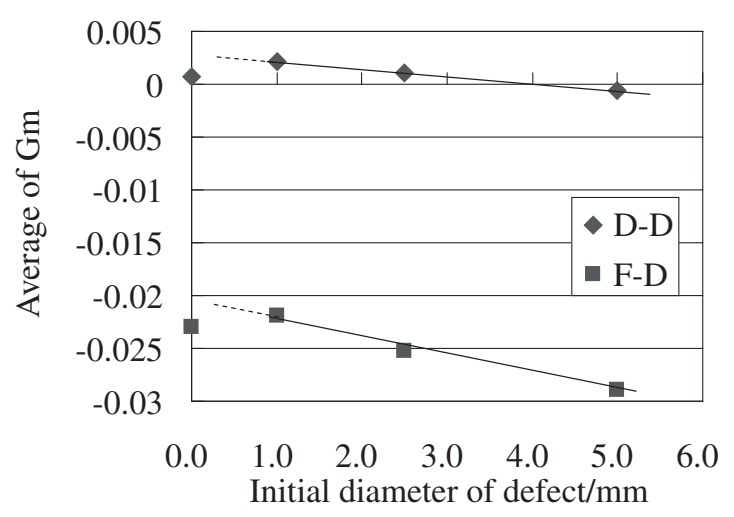

Fig. 16 Relationship between diameter of defect and average of Gm, $R e \fallingdotseq 9 \%$.

near a linear extension of the correlation between the defect diameter and the average of $\mathrm{Gm}$, although not coinciding perfectly with the extension of that correlation. On the other hand, regarding the absolute value of $\mathrm{Gm}$, verification of a closure effect by rolling, etc. of square blooms with flat rolls has been reported. ${ }^{23)}$ Because that report described the results of an analysis with solid materials, if the stress field is defined in the same manner as in the present report, the value of $R e$ of the reduction in area at around $9 \%$ is approximately -0.02 . Compared with a solid material, that is, with a defect diameter of $0.0 \mathrm{~mm}$, there is a difference between diamond rolling in which $\mathrm{Gm}$ is smaller, and flat-diamond rolling which shows a comparatively close value. It is suggested that the index related to Gm differs depending not only on this, i.e., on reduction conditions, but also on the rolling type, for example, the caliber shape, etc.

From the above, in cases where an asymmetrical tendency is remarkable, for example, when strong asymmetry exists in the rolling type, defect shape, etc., it is now clear that discussion of defect closure based only on the reduction in area of the bulk material is inadequate, and it is necessary to introduce a stress field such as Gm of the like, the application of which was studied in this work, and a parameter that can express the distribution of that stress field.

\section{Conclusion}

The stress distribution generated in the vicinity of a material center defect relating to closure of casting defects in the center of steel billets was studied of round billets of SUS420J1 having an artificial center defects with surrounding slits by experiments and numerical analyses using the rigid-plastic finite element method analytical solver CORMILL. Results are as follows.

(1) The stress field generated at the hole edge (artificial defect) was estimated from the opening/closing behavior of slits in diamond caliber rolling. The results showed good agreement with the stress field obtained from the FEM analysis.

(2) Results of numerical analyses using FEM solver CORMILL of a flat-diamond caliber rolling simulated accurately experimental results regarding relativity of the slit opening/closing behavior and hydrostatic pressure distribution, demonstrating that it is possible to estimate the hydrostatic pressure distribution, which strongly affects hole edge closure. This rolling type displays a distinctive asymmetrical stress distribution.

(3) When discussing defect closure using the hydrostatic integration parameter $\mathrm{Gm}$, under conditions of strong asymmetry, when the stress/strain distribution changes easily, this research demonstrated that analysis using a conventional solid specimen is not necessarily adequate, and it is necessary to apply a parameter which directly considers the effect of defects.

(4) Summarizing the above, in prediction of defect closure, the necessity of considering the stress distributions under respective rolling conditions was suggested.

\section{REFERENCES}

1) U. Ståhlberg, H. Keife, M. Lundberg and A. Melander: J. Mech. Work. Technol. 4 (1980) 51-63.

2) W. Johnson: Appl. Sci. Res. A 7 (1957) 65-88.

3) M. Kawai and K. Kamishobara: The 7th Int. Forg. Conf., Paris, Apr, (1975) pp. 571-596.

4) I. Tamura, S. Watanabe, K. Watanabe and K. Nakajima: Trans. ISIJ 24 (1984) 101-106.

5) S. L. Wang and Q. X. Cao: J. Mater. Process. Technol. 43 (1994) 195209.

6) S. L. Wang and Q. X. Cao: J. Mater. Process. Technol. 43 (1994) 211220.

7) M. Kawai and K. Sato: Int. Forgemasters Meet. 8th (1977) pp. 3(1)3(14).

8) A. Wallerö: J. Mech. Work. Technol. 12 (1985) 233-242.

9) A. Wang, P. F. Thomson and P. D. Hodgson: J. Mater. Process. Technol. 60 (1996) 95-102.

10) H. Tagawa, J. Tanaka, T. Hirawasa and E. Sunami: Tetsu-to-Hagane 62 (1976) 1720-1733 (in Japanese).

11) S. Tsuyama, S. Masuda, H. Tagawa, T. Hirasawa and H. Suzuki: Tetsuto-Hagane 71 (1985) 86-92 (in Japanese).

12) S. Ono, K. Minami, E. Murai and T. Iwadate: Trans. Jpn. Soc. Mech. Eng. C 62 (1996) 1139-1146 (in Japanese).

13) M. Kiuchi and S. Hsiang: Steel Res. 56 (1985) 261-269.

14) K. N. Shah, B. Kiefer and J. J. Gavigen: ASME Product. Eng. Div. (1986) pp. 47-61.

15) Z. Wang and M. Ren: Adv. Technol. Plast. 2 (1993) 1181-1186.

16) S. P. Dudra and Y. Im: Int. J. Mach. Tools Manufact. 30 (1990) 65-75.

17) M. Tanaka, S. Ono and M. Tsuneno: J. JSTP 27 (1986) 852-859 (in Japanese).

18) M. Tanaka, S. Ono and M. Tsuneno: J. JSTP 28 (1987) 238-244 (in Japanese).

19) X. Zhang, Z. Cui, W. Chen and Y. Li: J. Mater. Process. Technol. 209 (2009) 1950-1959.

20) U. Ståhlberg: J. Mech. Work. Technol. 13 (1986) 65-81.

21) R. I. Asfahani and J. R. Kahrs: Mech. Work. Steel Process. XXXI (1993) 371-378

22) S. Ono, K. Minami, T. Ochiai, T. Iwadate and S. Nakata: Trans. Jpn. Soc. Mech. Eng. C 61 (1995) 2141-2146 (in Japanese).

23) M. Nakasaki, I. Takasu and H. Utsunomiya: Tetsu-to-Hagane 94 (2008) 284-289 (in Japanese).

24) M. Oyane: J. Japan Soc. Mech. Eng. 15 (1972) 1507-1513.

25) T. Iguchi, H. Hayashi and M. Yarita: J. JSTP 35 (1994) 959-964 (in Japanese).

26) J. Yanagimoto, M. Kiuchi, M. Nakamura and T. Kurahashi: J. JSTP 32 (1991) 1000-1006 (in Japanese).

27) K. Inoue: Tetsu-to-Hagane 41 (1955) 593-601 (in Japanese).

28) K. Misaka and Y. Yoshimoto: J. JSTP 8 (1967) 414-422 (in Japanese).

29) Y. Saito, Y. Takahashi and K. Kato: Tetsu-to-Hagane 64 (1978) 250259 (in Japanese). 